\title{
Article \\ STEP-NC based squashing slicing algorithm for multi-material and multi-directional additive process
}

\author{
Jumyung Um ${ }^{1, *}$,Joung min Park ${ }^{1}$, Ian Anthony Stroud ${ }^{2}$ \\ 1 Kyung Hee University, 1732 Deogyeong-daero, Yongin-si, Republic of Korea ${ }^{2}$ SkAD Labs SA, Chemin de \\ la Raye 13, 1024 Ecublens, Switzerland \\ * Correspondence: jayum@khu.ac.kr; Tel.: +82-31-201-3695
}

\begin{abstract}
The paper describes problems with the current additive manufacturing chain before considering additive manufacturing as part of a modern manufacturing chain. Additive manufacturing can be used for near net-shape for finishing, for repair or for adding special features which cannot be made with metal cutting. Recently ISO TC184/SC1/WG7 published ISO 14649-17 for proess data of additive manufacturing. This paper describes how STEP-NC deals with these different scenarios in terms of accuracy, multi-material and variation of slice direction. The possibilities of multi-material objects also raises questions about the design of such objects and how these need to be handled by an advanced controller. The paper also describes non-planar slicing. Curved direction and cylindrical direction are shown to improve the accuracy of curved structure additive manufacturing. STEP-NC using boundary representation has better capability of depicting complex internal structures for additive processes. By using exact model of the final product represented by STEP-NC, the paper demonstrates improvements in data size reduction, slicing accuracy, and precise manipulation of internal structure.
\end{abstract}

Keywords: Additive manufacturing, STEP-NC, Boundary representation, Multi-materials

Citation: Lastname, F.; Lastname, F.; Lastname, F. Title. Preprints 2021, 1, 0. https://doi.org/

Received:

Accepted:

Published:

Publisher's Note: MDPI stays neutral with regard to jurisdictional claims in published maps and institutional affiliations.

\section{Introduction}

Additive manufacturing has been the focus of much research over the last decade and is considered one of the major upcoming production processes which will be used for new practical manufacturing. This paper describes geometric processing to derive final sections utilised by an additive machine controller directly from a CAD model. Before this, however, it is necessary to say something about the additive manufacturing process chain. This chain is well known. That is to say, there is a common assumption that the chain is well-known, but there are a number of variables that are less well understood. The common view of the chain from CAD to part is:

1. CAD model

2. Approximation as STL (or maybe AMF)

3. Transfer to pre-processing software and correction, if necessary

4. Slicing and offsetting

5. Generating supports, if necessary, and tool path

6. Slice transfer to additive machine

7. Part manufacture

Rather than being planned, this chain has been established by practice. Before describing the modern, planned chain determined by Bonnard [1] it is worthwhile reviewing the normally assumed chain origins.

AM (Additive Manufacturing) machines started to appear at about the same time as graphics computers, forerunners to graphics cards. In order to understand why the STL (Stereolithography) format has been used for communication of solid data, it is necessary to look back at this period. At that time there were two main techniques for representing solids, CSG (Constructive Solid Geometry) and BRep (Boundary Representation). Although these two uses very different methods for representation, the CSG method generated a 
BRep model for graphics because direct graphics from CSG models proved too slow. So, the boundary of a solid, essentially the "skin" between the inside and outside of a solid model was a common factor. However, there was then no agreed format for communicating boundary data to applications. Another solid modelling method, the Octree method, was used for communicating volumetric data but eventually the skin of the object, communicating using triangles, gained dominance as a graphics communication format.

The idea of producing a real physical model as a visualisation tool may have encouraged the use of triangles, in the form of an STL file [2], as a means of communication for additive manufacturing. However, the STL format is, and always has been, a format for graphics, not for communicating solid information, which is what is needed for additive processes. For graphics purposes it is unimportant that the triangles are unconnected, but for additive processes it is critical that the triangles match and that the whole set is closed. In graphics the triangles are used to colour parts of the screen, so connectivity is unimportant and visual gaps only an annoyance. Nor is the shape of the triangle critical because the normal vector is given explicitly and does not need to be calculated from the facet vertex positions, so avoiding the problem of degeneracy. However, slicing triangles can lead to numerical problems if they are long and thin. In addition, faceting of surface models which may not be closed and separate faceting of faces in solid models leads to problems of mismatched facets, again not a problem for graphics but a major problem for additive manufacturing.

Dissatisfaction with STL has long been apparent. In 1994 Carleberg [3] suggested using STEP [4] for shape communication. Kumar and Dutta KuDu1997 produced a survey of different formats. However, despite the problems of STL, its use has continued unabated. The new suggestion, AMF (Additive Manufacturing Format) [5] corrects some of the shortcomings, adding material and colour information, but is still, essentially, a graphics format, unsuited for communicating solid model data.

In the meantime, since the original additive machines appeared for 3D printing of solids, the use case of additive manufacturing has changed. Traditional use case, 3D visualisation models and prototype manufacture additive processes have moved into real manufacturing with moulds (rapid tooling) and, as processes improved, for manufacture of real parts, repaired parts, and re-furbished parts. Even though the use case has broadened, mass production is still a challenge to AM processes because of lack of accuracy and the physical realities of additive processes. Most additive parts with curved or sloping surfaces have a characteristic step-wise surface roughness because models are sliced to produce layer shapes which are extruded in a single direction. Post-processing operations, such as grinding process, solvent immersion, EDM (Electrical Discharge Machining) subtraction, improve the surface accuracy but, only when the parts are slightly larger than the required shape. It is not guaranteed in the normal chain because missing volume creates problems. Finally, the data format used for AM processes is a tessellated model which means increasing the size of the data, if accuracy needs to be high.

The first serious examination of the needs of manufacturing was performed by Renan Bonnard, then at the Ecole Centrale de Nantes[1]. His work led to the development of the STEP-NC standard for additive processes which defines a consistent format for additive processes as part of a manufacturing chain. The data model [6], the environmental aspects [6], hierarchical structure [7] are investigated as the following research. Additive parts almost certainly have to be finished with material removal processes in parts in order to achieve the quality expected in manufacturing. Um et al.[8] proved the advantage of using STEP-NC in AM processes as part of remanufacturing.

As well as remanufacturing, additive manufacturing is widely utilised for more complex applications. This means that AM is considered as one of production process in the whole supply chain. Hybrid manufacturing, which combines subtractive processes 
and additive processes, has appeared in commercial machine tools and requirements for mixed process planning are discussed by Newman et al.[9]. They also present the need for STEP-NC in additive manufacturing. Medical products, aircraft, racing cars and dental work are recent applications of additive manufacturing, but STEP-NC does not cover all AM specialised operations and features [10][11]. Various application industries, as well as typical manufacturing industry, utilise AM, as mentioned by Lee [12]. Advanced production technology, such as $4 \mathrm{D}$ printing, has also been launched[13].

This paper proposes a novel data representation based on STEP-NC and a new geometric procedure for producing slice geometry. In section 2 the basic concept and existing model for this paper are introduced. Section 3 describes new features and representation of AM features based on the STEP-NC representation. Slicing operations and a way to ensure that volume is added for post-processing by machine tools follows in section 4 . Section 5 explains the result of experiments with several shapes. In section 6 , the difference between the approach presented and conventional models is described, with a discussion of results in section 7. Conclusions are given in Section 8.

\section{Multi-material Additive manufacturing}

Even though many existing data formats have been developed, there is still a gap to satisfy the requirements of improving geometric processing. Software systems for additive process require high slice density, multi material data format, and fine slicing operations in order to produce accurate shape. 1) In this section, data formats which are used are investigated from STL [2] to STEP-NC [14] and AMF [5], recently established as an international standard. Then, 2) existing data representation formats for additive manufacturing which are specifically for multi-material processes are discussed. Some representations and strategies for multi-material structures, a key function for slicing whole shapes into fractional pieces, are introduced from aspects of improving the accuracy of the final output shape. Also, 3) special treatment of slicing operations area, applied to increase the efficiency of handling complex geometry.

\subsection{New requirements of multi-materials in Additive manufacturing}

Along with the advance of additive machine tools, multi-material representations are required for the upcoming CAD/ CAM/ CNC chain for additive manufacturing. To fulfil the demands, there are existing efforts to depict the complexity of free-form surface and internal structures. Multiple material additive manufacturing is reviewed by Vaezi et al.[15]. They also found inherent limitations for multiple material printing and addressed the necessity for hybrid systems.

Kokkinis et al.[16] proposed another usage of additive processes to produce multiplematerial and shape-changing objects manipulated by magnetic control. Pa et al.[17] also produced low-profile antennas with multi-material additive manufacturing. Ge et al.[18] established the concept of "4D printing" with tailorable shape memory polymers and emphasised the necessity for simulation models. Liu et al.[19] addressed the quality issues of multi-material surfaces for bonding.

Gupta and Tandon[20] proposed the gradient representation of multiple material and multiple colour of additive process.

The new STEP-NC part (ISO 14649-17) for additive processes has the freedom to use many representations from simple lines to NURBS surfaces. The collection of representations is the source of a new data format of additive process.

\subsection{Evolution of Data representation}

A survey of data formats for layered manufacturing was carried out by Kumar and Dutta[21] in 1997. It has long been recognised that improving the accuracy of handling geometric data requires a new representation format even though STL has been the de-facto format used for communicating shapes in additive manufacturing. It should be pointed out that there is no technical necessity for using either STL or any other facetted format. 
Slicing of exact Boundary Representation models was developed at the end of the 1970s in the BUILD system, though not then for additive manufacturing. Other slicing algorithms were developed in the 1980s and slicing exact models were a function for engineering drawings in CAD systems. The suggestion by Carleberg[3] of using STEP when that became a standard for solid model data exchange was logical and realistic. Pratt et al.[22] examined the issue of the accuracy of the STL representation in rapid prototyping during data transfer through the CAD-CAM-CNC chain and emphasised the usage of the STEP representation. Hiller and Lipson proposed a new data format representing the geometry with a triangulation based express format called AMF (Additive Manufacturing Format) to improve the accuracy from a flat triangle representation [5]. As well as data representation, it is necessary to improve geometric operations such as scanning and printing direction to maintain seamless representation accuracy. Wang et al.[23] proposed an optimisation method for printing direction to improve surface quality. Mohammed et al.[24] investigated additive manufacturing in prosthetic rehabilitation. Their work used scanning to construct the geometry of the relevant body area, for them the face, design and finally additive manufacturing to create the prosthesis.

\subsection{Necessity of exact model in additive manufacturing}

The last piece of work mentioned above illustrates a difference in the use case of additive manufacturing. For mechanical parts there is usually an exact model available, created using a CAD system. For aesthetic design parts scanned from real shape for example, the 'model' can be either an agglomeration of points through which to fit surfaces or a file in STL format. It can be argued that it is unnecessary to create exact surfaces and that the use of a set of triangles together with manual finishing based on human aesthetic judgement is more efficient for design parts.

Similar considerations can be made about industrial design parts with aesthetic forms. In mechanical parts where the exact geometry exists, conversion into a facetted form such as STL or AMF is inefficient. Geometric intersections are stable and available through commercial and open source kernel systems. They are more efficient than intersecting a lot of triangles and numerically more stable.

Another important consideration is the stage when the geometry data is approximated. The ideal case is that all shape information is passing seamlessly until the numerical controller of additive machine. And numerical controller directly generates approximated curve paths in the last minute. On the other hand, using the approximated model in early design stage causes the accumulation of the error made by the series of geometric processes. Best case is to reach the accuracy of numerical controller by Using an exact model for efficiency and accuracy.

The STEP-NC format, see Bonnard et al.[25] under standardisation by ISO TC184/SC1/WG7 as ISO 14649-17, allows communication of an exact model for direct control. The format also allows post-processing, if required by the manufacturer, to either STL or AMF with parameters determined from user requirements. Also Bonnard et al.[26] described that the two formats STL and AMF are enablers for rapid evolution but not suitable for delicate manufacturing. The STEP-NC format is also more appropriate if manufacture switched between machines with different characteristics. Since STEP-NC records what is commonly termed a micro process-plan it is also suitable for archiving where the process parameters are recorded explicitly. Zhao et al.[27] explained by using STEP-NC formats advantage, richness of information, are also more proper format for maching simulation. For further considerations, see Um et al.[8] which compares existing data representations with the STEP-NC data representation in an additive manufacturing process for re-manufacturing.

\subsection{Calculation of added volume}

Surface quality insufficient for final production was discussed by Carleberg in terms of product modelling dimension manufacturing [3]. Generating added volume is the next target to be improved for high quality and robust output of additive process. Special 
treatment of slicing operations are also applied to increase the efficiency of handling complex geometry. This kind of treatment does not exist in subtractive processes. Slicing methods have been developed to enhance the surface roughness and part strength of the final part. Overhang and undercut are serious problems disrupting the output quality. The slicing algorithm for a 5-axis table is discussed by Lee and Jee [28]. Physical weakness is addressed and a modified slicing algorithm was proposed by Steuben et al. [29]. In terms of the material usage, topological optimisation is applied to additive manufacturing to reduce the amount of support structures generated after an additive volume is created. Wu et al.[30] proposed an application based specific infill structure algorithm to optimise added/removed interior supporting structures. Changing the direction is also applied in Voronoi cell structure, see Wang et al.[23].

\section{Data representation}

"How should the target objects be designed" is the question to legacy CAM software for additive processes. Using STEP-NC is a key way for deriving exact slices to fit the final shape the designer wants. ISO 14649-17 is the standard of STEP-NC for additive processes and is integrated with the other ISO 14649 parts for hybrid process plans. The intention of this section is twofold, 1) Firstly, STEP-NC data format is introduced with the comparison to voxelisation method. 2) Secondly, gradient boundary representation generated by various source types is introduced to describe multiple material or multiple colour object. Finally, we discuss how to apply both features to the build-up phase.

\subsection{STEP-NC standards}

Multiple materials structure and complex structures inside the product are also applications of additive manufacturing. The ways in which these shapes are expressed is broadly used in tessellation or in the Voxelisation method. Voxelisation methods generally make the data size larger depending on the shape complexity, as with point clouds. It also has the disadvantage of not having the high resolution of the original features once converted. BRep is a way of expressing complex shape accurately with smaller capacity compared with voxels or triangles, as well as direct inputs of the squash operation which can produce accurate and high-quality results.

In the case of STEP-NC, additive features present some ways of expressing BRep based representation. Figure 1 shows the data structure for STEP-NC additive manufacturing. Based on part 10 of the existing STEP-NC, the following definitions were made for AM following the definitions for milling and turning. First, the features used in STEPNC are: 1) am_compound_feature representing multiple added features in compound information structure, 2) am_gradient_feature allowing graded materials and colours produced by additive process, and 3) am _heterogeneous_feature defined by a mathematical function adjusting heterogeneous materials and colours. The operations consist of: 1) am _oned_operation which produces one filament to obtain the full geometry, and 2) am_twod_operation defining the attributes of guiding geometry, thickness and normal vector.

\subsection{Multi-material objects}

It is not yet clear how multi-material objects should be designed. This is not a subject for this paper but it would be helpful for manufacturing to have an insight into how the object was designed. In the meantime, other methods for defining multi-material objects have been sought.

Two approaches for multi-material objects are to use explicit hard boundaries using graded materials where the material properties are calculated in some way or using voxel based representations. 


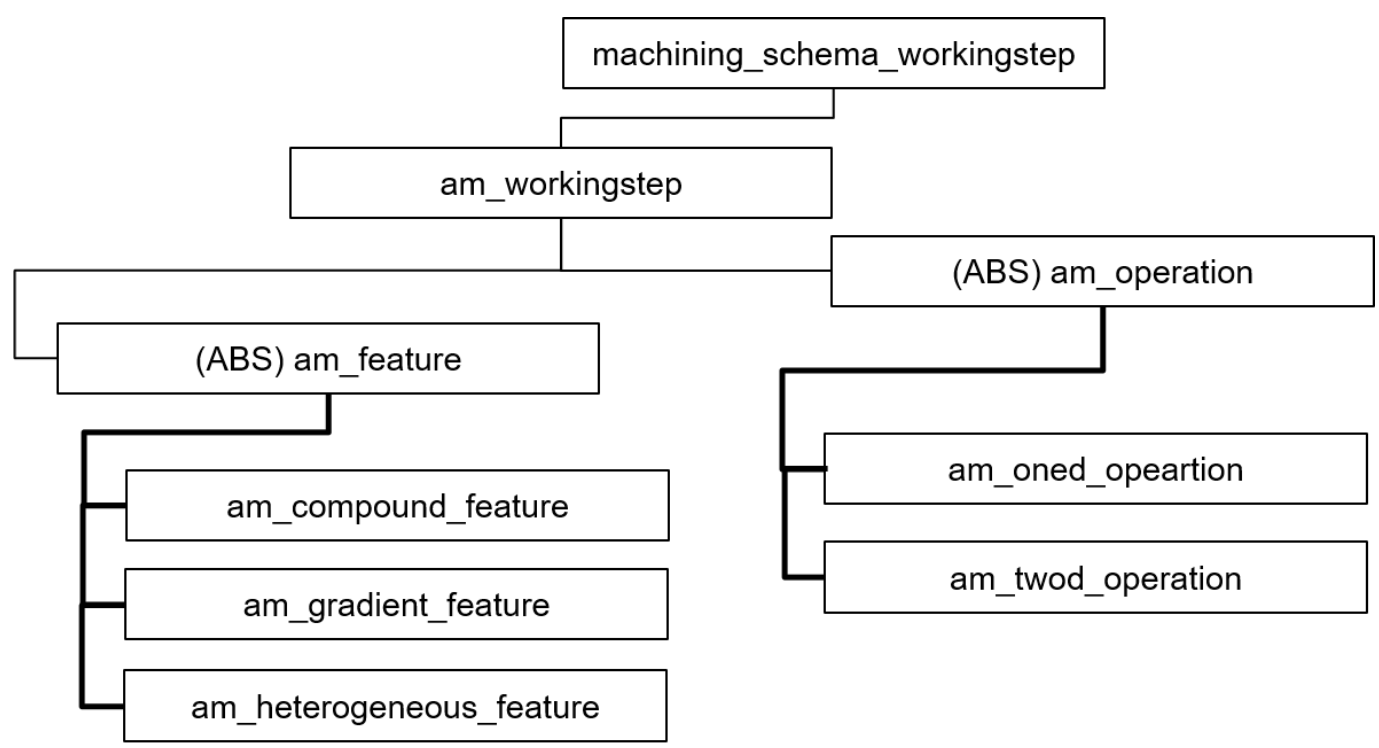

Figure 1. The high-level structure of STEP-NC additive manufacturing.

For explicit boundaries, the structure can be described using Boundary Representation, BRep, with non-manifold internal boundaries. However, there are some considerations. Unless the internal boundaries are always aligned with the build direction, somewhat unlikely, the squashing process will define overlapping areas where there are two materials defined. This can be resolved by using grading techniques or by giving material definitions priorities so that one material will be chosen over another in the overlapping region. An interesting corollary to this is that holes can be defined as internal structures having an 'empty' material. If that empty material has a higher priority than other materials then this will force the hole to be open, although it may be bigger than designed. In other cases if the hole is oblique compared to the build direction then the squashing process will tend to make holes smaller. The problems of holes growing or diminishing in size is a side effect of the discrete nature of additive processes and has to be taken into account both by designers and manufacturing planners.

Another method of representing multi-material objects is to provide a functional definition using which material properties at a given point that could be calculated. An example of this is shown in Figure 2 using two surfaces as a guide for calculation.

Voxel-based methods use a cellular structure with explicit material properties assigned to each cell. There are known disadvantages of using voxel-based cellular representations. One is that the precision of the representation depends on the cell size, higher precision demanding more memory, so the representation is scale dependent. Another disadvantage is that the material properties of each cell are absolute, leading to hard internal boundaries rather than smooth grading. Voxel-based representations are, to some extent, attractive because of their simplicity and clear intention. However, it is not practical to expect users to define cell material properties explicitly for each cell. Instead, some sort of functional approach to material definition is realistic, but then this method could be defined in either BRep or the functional gradient approach.

For example, a BRep internal structure can use the ratio formulation, as shown in Figure 3 , and requires a limited number of parameters to draw the material distribution.

In addition to the polynomial or linear functions, it is possible to use NURBS geometry to create internal patterns. Figure 4 shows a formulated model between two boundary curves having the relationship of gradient distribution from $l_{1}$ to $l_{2}$. There is a correlation between each pair of vertices $v_{1 i}$ and $v_{2 i}$. A NURBS formulation is applied along the line $R_{i}$. In order to define the distribution, the input parameters are knot vectors, degrees, and control points to configure the distribution in multiple dimensions. NURBS also allows 


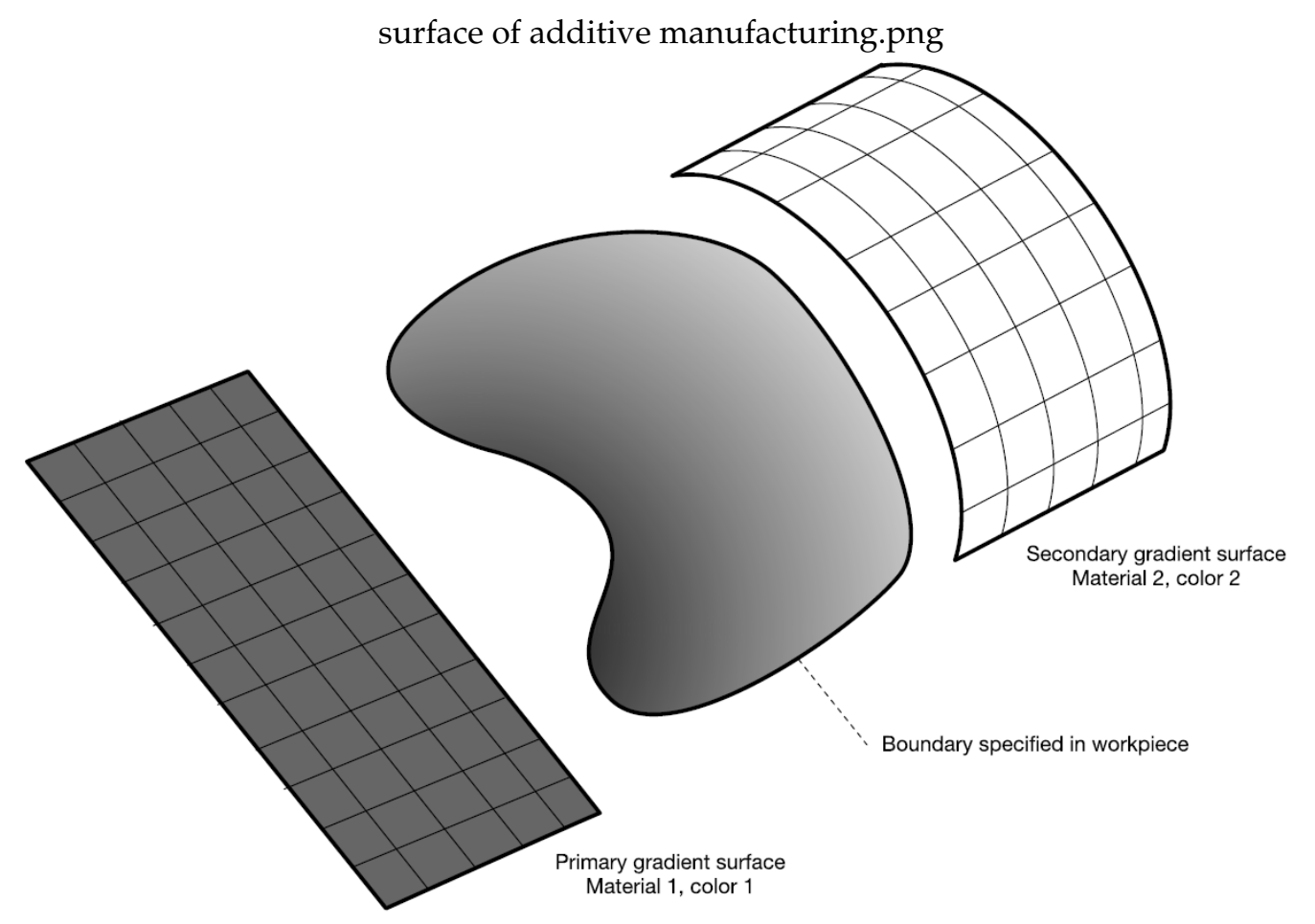

Figure 2. Gradient surface of additive manufacturing.

for multi-dimensional space in one-dimensional two-dimensional space, as well as more accurate representation of a given feature. In the slice operation, it is necessary to determine the colours for each slice and each path. Figure 5 depicts the colour allocation of each slice (upper) and of each path (bottom).

\section{Slicing operation}

\subsection{Approximation Slicing}

The approximation depends on the distance of facets to the real surface rather than on the layered cross-sections. This means that it is likely that the corner points of planar sections do not actually lie on the real surface but on approximating facets inside or outside the real body. The result is a slight warping of the intended shape, possibly not noticeable to the eye but significant in terms of manufacturing precision.

An obvious first step is to use real geometry of parts, rather than approximation. This, too, leads to problems in getting the desired shape. The slicing problems are illustrated in Figure 6 and Figure 7. A common characteristic in both Figure 6 and Figure 7 is that the made shape lies inside the desired shape as the object expands and outside when the object is contracting, as indicated when comparing the red rings with the slices in both figures. In Figure 6, there is a representation of what might happen with a sphere. The right-hand side of the figure shows what might be the sections, the red ring indicates the shape intended.

As with the approximated shape in Figure 6, the first section misses the body, since the intersection is at a single point. This means that the shape made is slightly higher than the desired shape. The yellow shape on the left represents a cross section through a sphere approximated by triangular facets.

It is also necessary to point out that the accuracy of surface approximation with facets is commonly controlled by a chord-height parameter. This controls the maximum allowable discrepancy from the real surface. However, this is in the direction of the local surface normal. Single directional additive manufacturing adds slices in an arbitrary direction irrespective of the real surface normal. This means that there is no guarantee that the solid created will respect the specified chord height tolerance. 
a
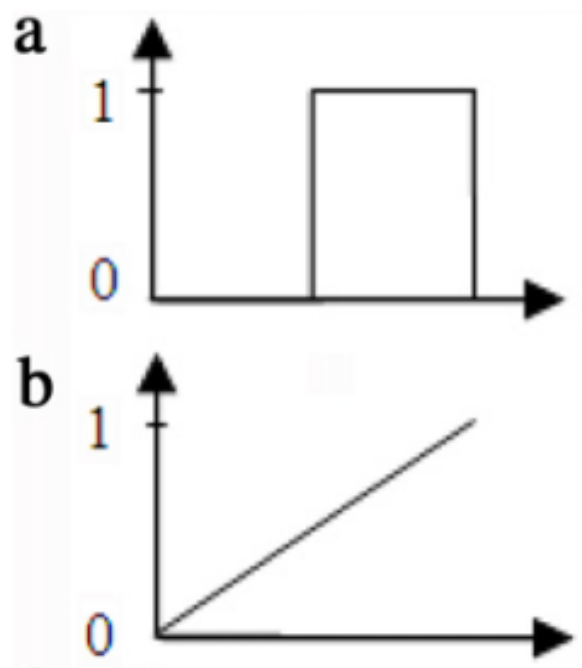

c
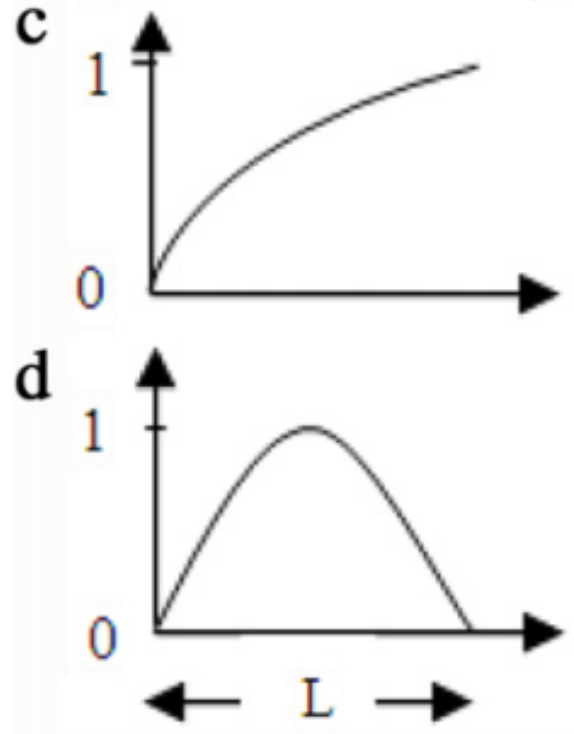
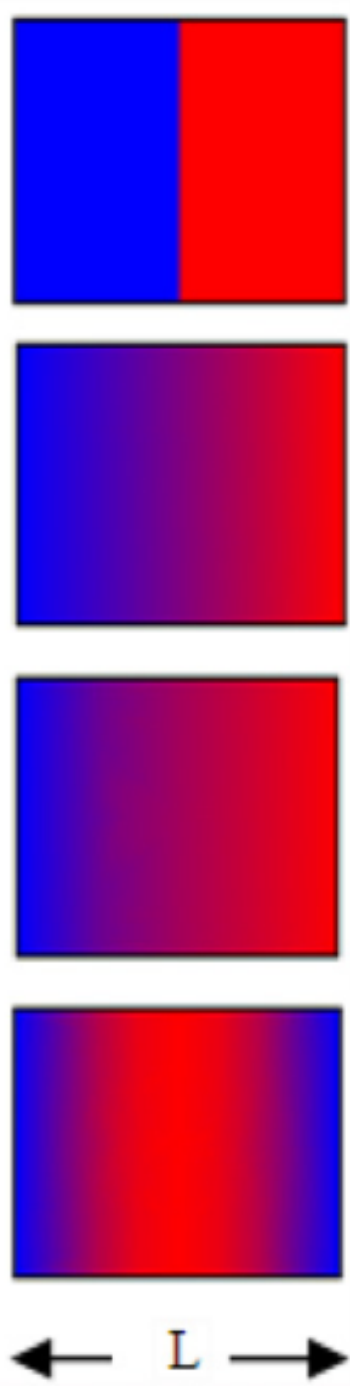

Figure 3. Two boundary curves[20].

boundary curves.png

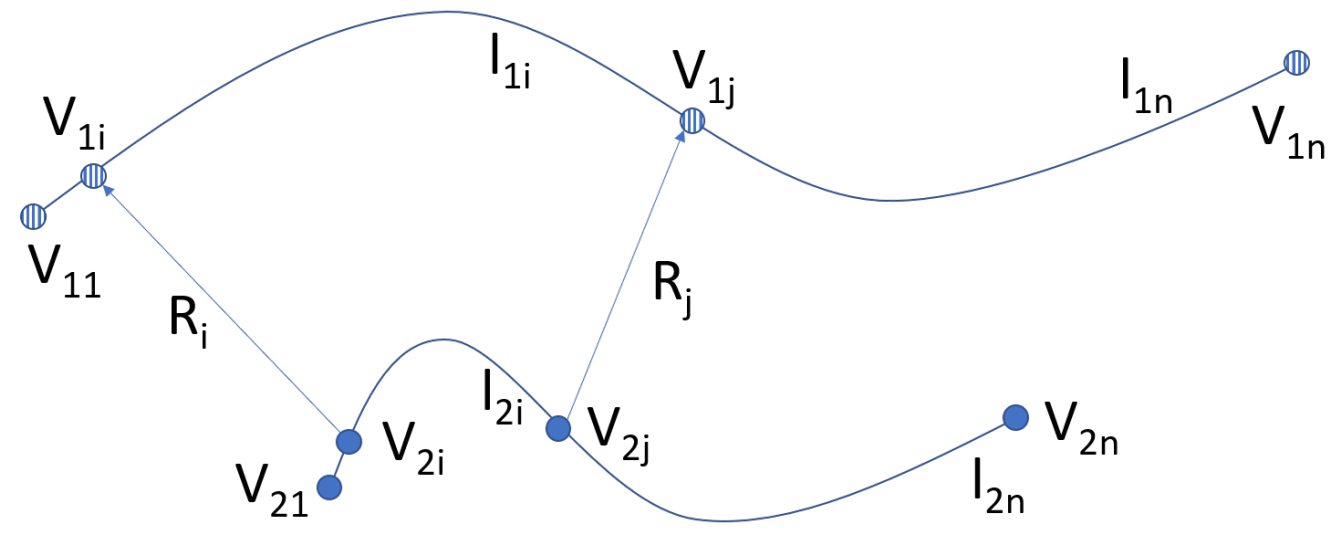

Figure 4. The notation of gradient distribution of two boundary curves.

This is simple geometric reasoning and fairly obvious. It is, however, important when considering additive manufacturing as part of a manufacturing process chain to make parts, where tolerance requirements are stricter than with visualisation. As indicated by the 
two curves 2.png
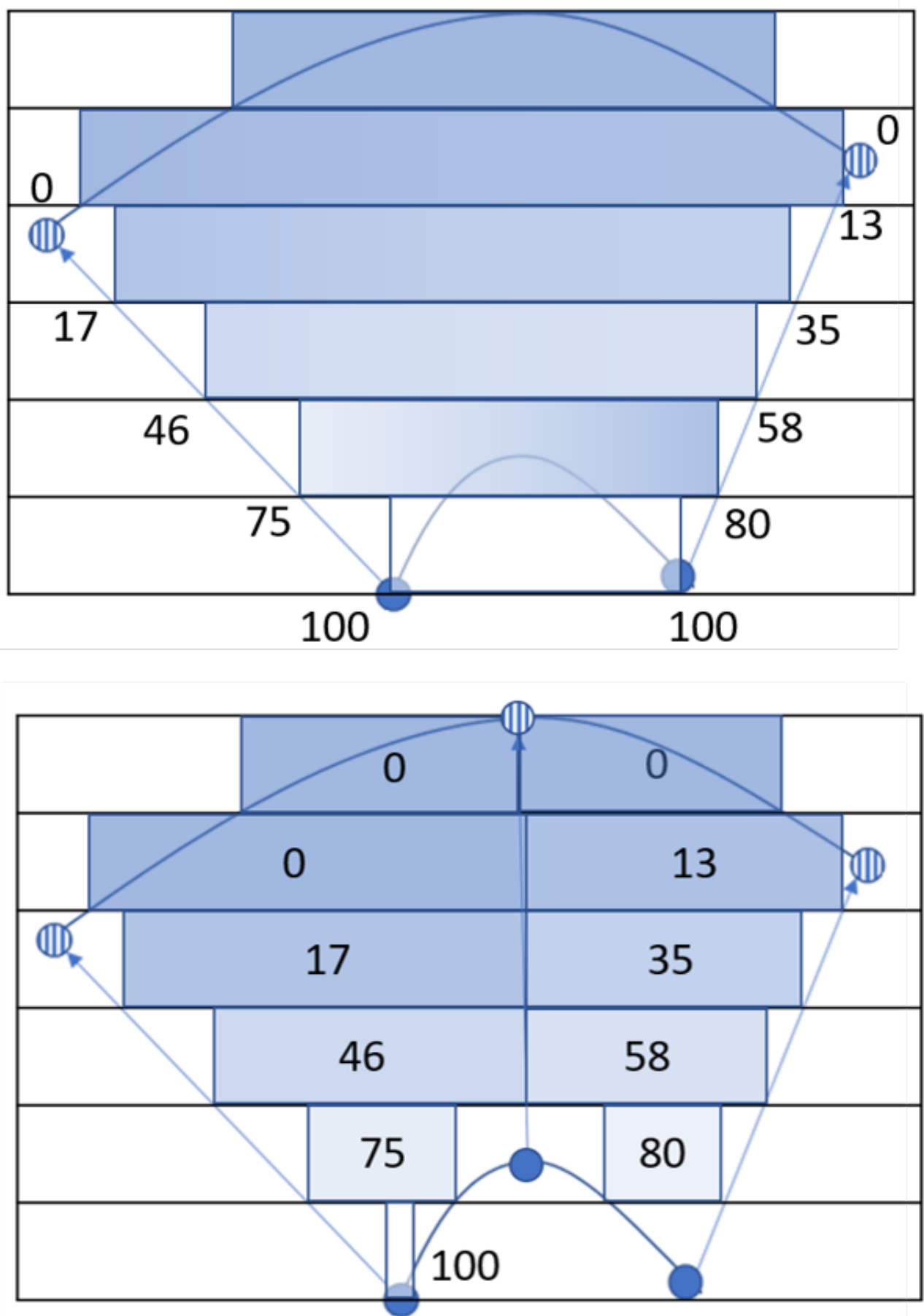

Figure 5. Slicing two curves.

original use of additive processes, and the currently popular name of 3D printing, additive processes for visualisation have much lower requirements than for manufacturing and hence the need for a different control protocol.

For manufacturing, additive processes should be seen as part of a multi-process chain. Because of the nature of additive processes, surface finish and precision are lower and so, for critical parts of a piece, it is necessary to finish by machining. This implies that the additive part should be slightly larger than the desired part all over. In order to achieve 

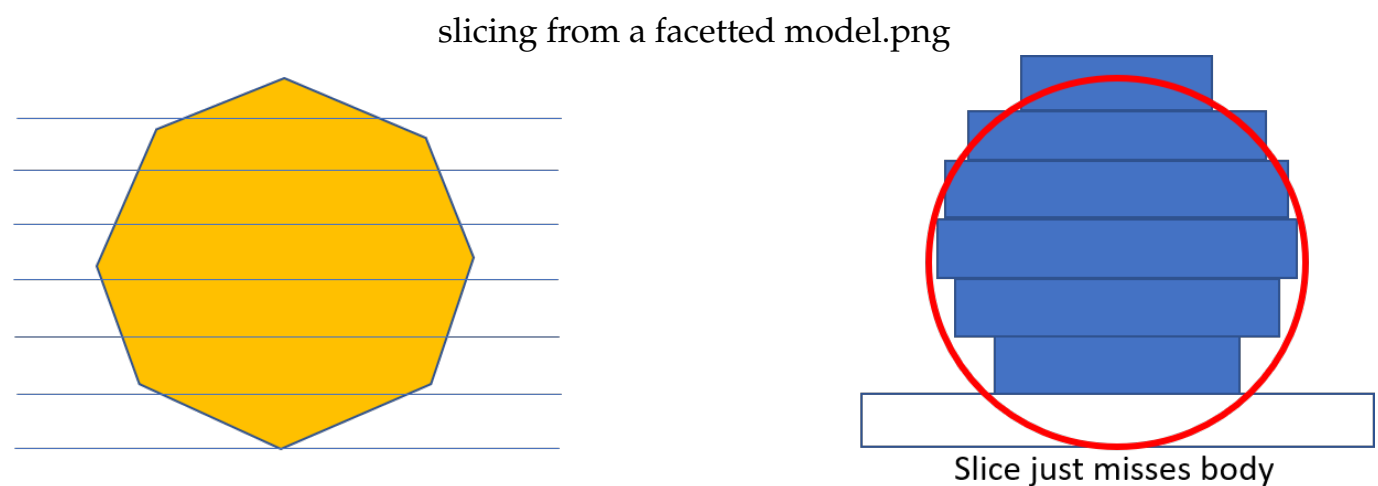

Figure 6. Naive slicing from a facetted model.

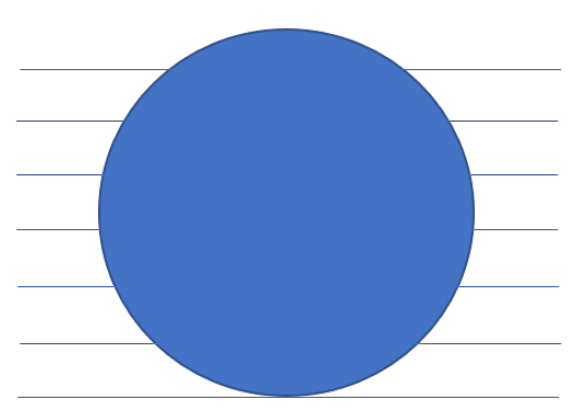

slicing.png

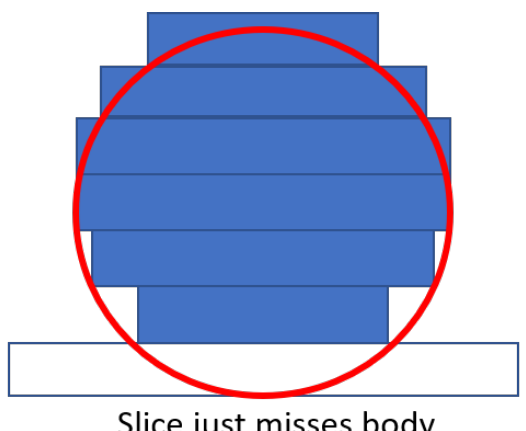

Figure 7. Naive slicing.

slicing with thick sliced.png
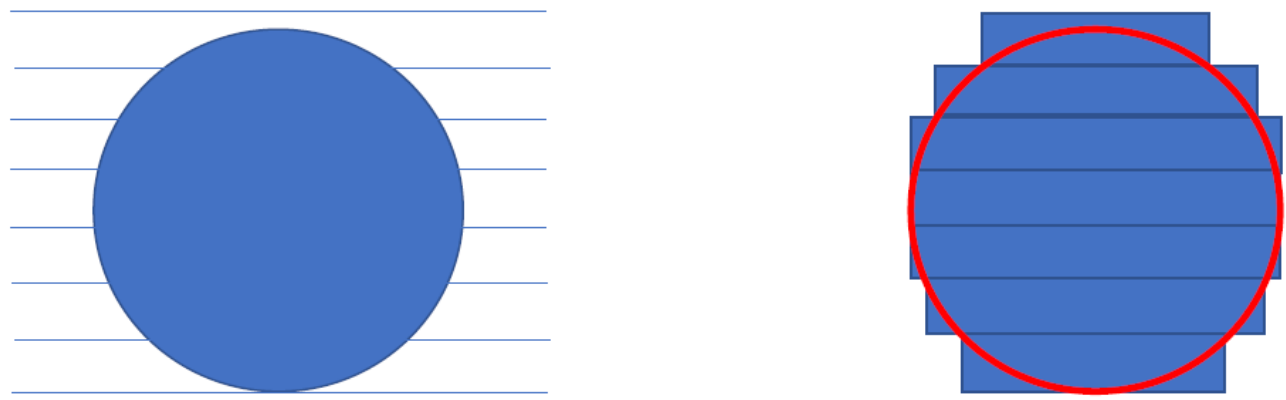

Figure 8. Exact slicing with thick sliced.

this it is necessary to determine the part footprint in each layer in order to determine the slice profile. Instead of taking a single slice, the object is sliced below and above the section to be produced, in effect an intersection with a rectangular block of thickness equal to the layer thickness, and this fat section squashed. The result would be as shown on Figure 8 , with material all-round the red-cross section.

Example of problems with naive slicing is shown in Figure 9. The desired object is shown at the top of Figure 9. The naive slicing, with a single slice profile is shown underneath. On the third row you have the result of additive with these simple slices. Depending on the angle of the slope, these elements may not even join and the whole sloping element would disappear. Even if they do join, the part thickness would diminish, possibly causing structural failure. With the thick sections you would obtain the object at the bottom which could then be finished by machining, if appropriate. 


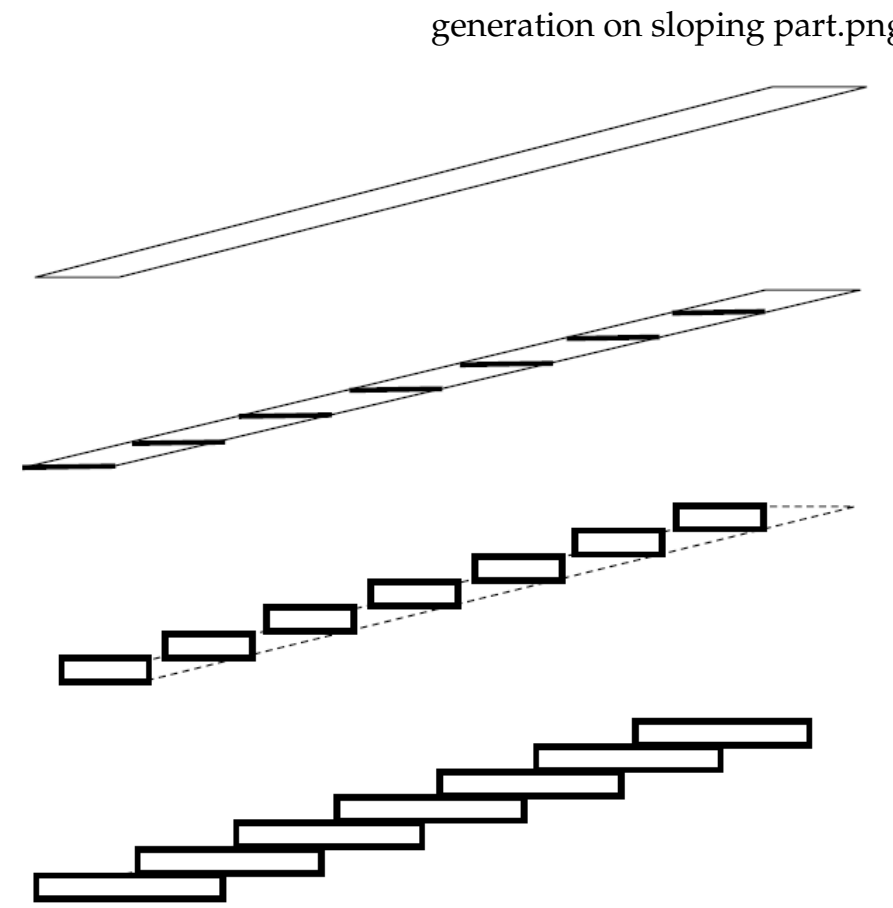

Desired object

Naïve slicing

Additive process

Object needed for manufacturing

Figure 9. Slice generation on sloping part.

The inaccuracy issues described above need to be resolved before machine tools start to build the final shape. Near-net-shape is required during geometric transformation to build the tool path for a numerical controller. Squashing is a way to extract Near-net-shape profiles and can be likened to making a pencil drawing around a fat section of the object laid on the floor. This is the silhouette of the fat slice viewed from the build direction. Each layer profile is projected onto the bottom plane and extruded along the identical direction with other slices. Squashing is similar to the common projection operation of solid kernel in terms of the range of Silhouette extraction from the cross-section of the designed shape.

\section{Squashing operation of specialised slicing}

\section{1. $2 D$ operation and process directions}

Part 10 of STEP-NC, or ISO 14649-10, defines the boundary representation entities and other parts for different processes. Additive processes as well as milling process and turning process have process specific definitions in other parts. In Part 10, technological entities defining process parameters also cover some of those parameters needed for additive processes. Figure 10 illustrates a gradient that shows the distribution between two grid planes. By fitting parameters, NURBS can represent arbitrary distribution of internal structures captured by scanners or CT imaging. Different types of feature can also be created in the form of core profiles.

Another way to define internal patterns as core profiles is to define the shape of slices according to the operation. Figure 10 shows three different regular slice geometries. The 'traditional' slice geometry is a plane. However, it is easy to imagine specialised machines to build curved or rotational parts, in which case different slicing geometries being useful. The most general is the cladding process which adds material to an existing object. In that case, it should be expected that a five axis machine needs to be used to apply material in general shapes. The shape of the 'slice' and paths then become general and geometric forms such as NURBS are needed.

\subsection{Slicing algorithm for special features}

In the manufacturing process, the slicing algorithm is the most important operation to maintain accuracy of the AM process. The algorithm described here uses a squashing 
opeartions of additive manufacturing.png
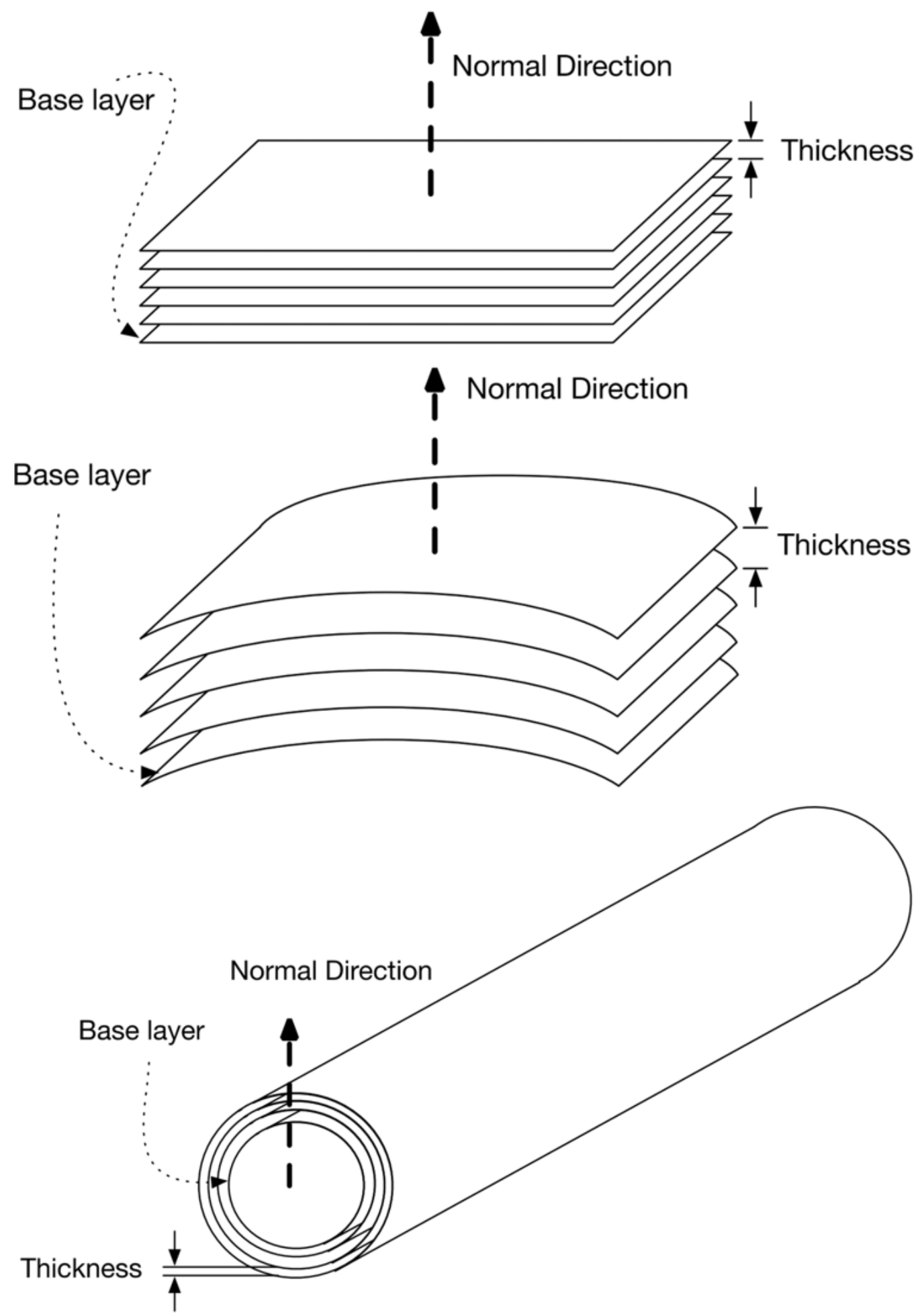

Figure 10. 2D operations of additive manufacturing.

operation to reduce the amount of gaps caused by the fluctuation of accumulated approximated sliced layers. This algorithm is able to keep fixed tolerance allowance and manage the empty space defined by the designer.

Gradient interpolation is conducted during the slicing process. It does not use the approximated representation data format in design phase but performs last minute modification to maximise accuracy in machine tool performance. The algorithm for building a 


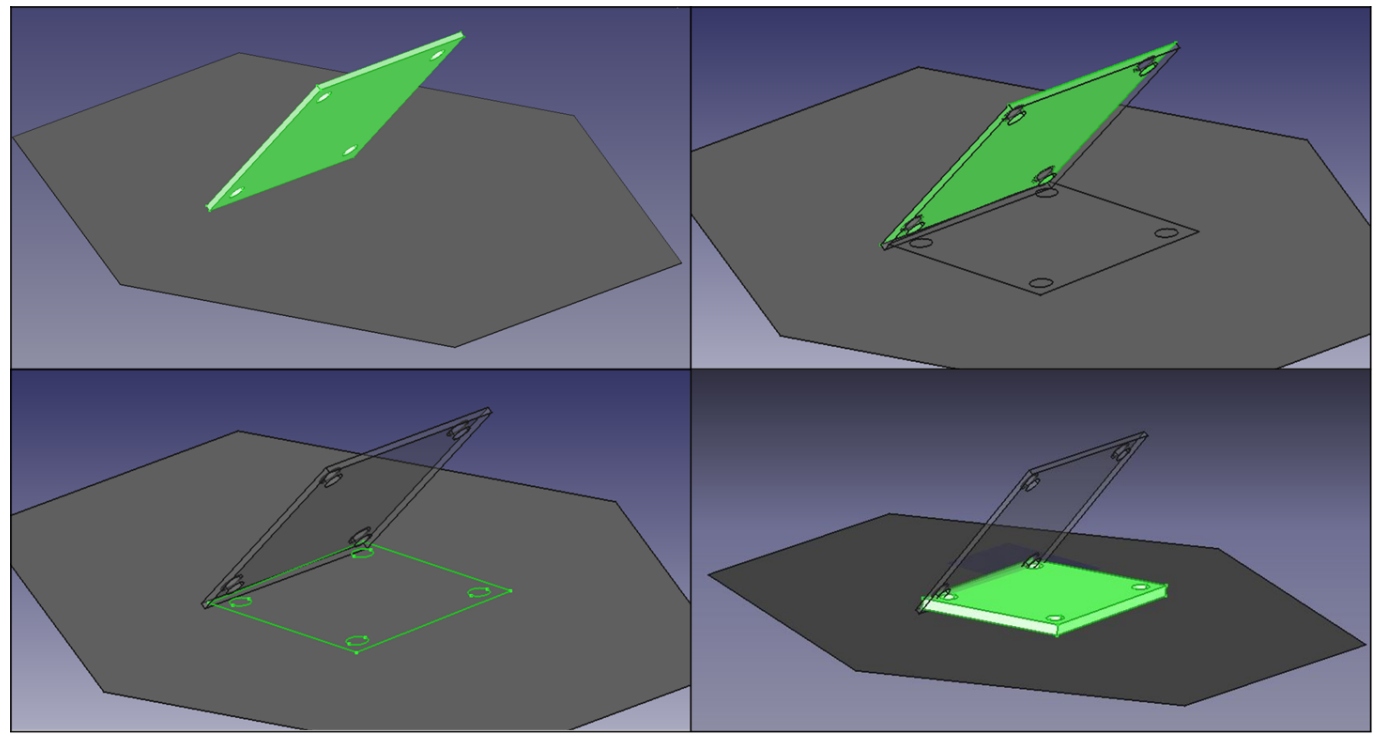

Figure 11. Procedure of squashing operation on single slice.

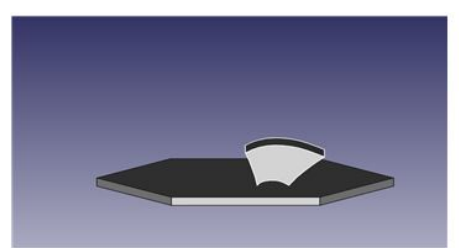

(a)

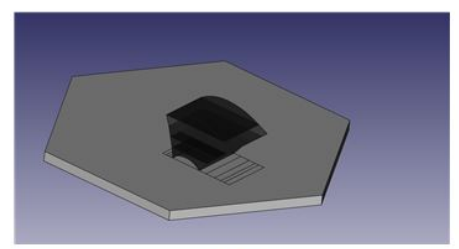

(d)

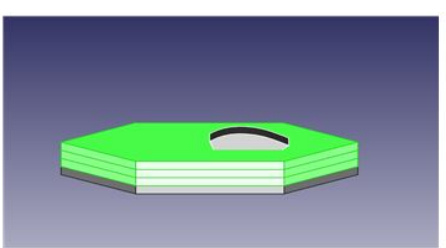

(b)

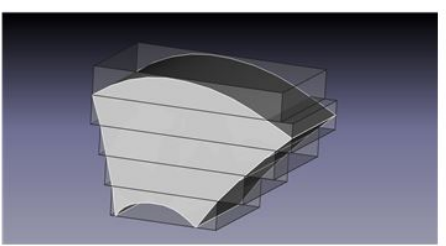

(e)

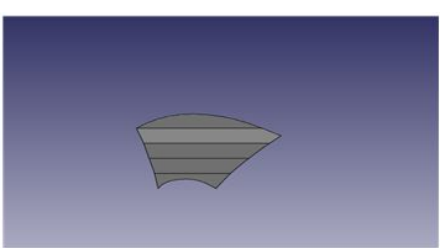

(c)

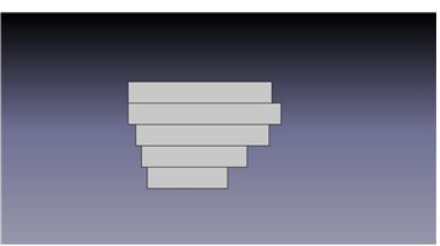

(f)

Figure. 12. Squashing Operation Process. Duplicate solid plane by V direction, (c) Intersection part, (d) Projection,

(e) Extrusion, (f)Final Shape

squashing operation consists of five stages: 1) First, definition of the slicing plane which, with the layer thickness defines the slicing block. 2) Next, intersection using a Boolean operation between the slicing block and the desired object to calculate the actual slicing geometry of the layer. 3) Projection of all edges in the calculated slice along the slicing direction of each layer. Each projected edge becomes a portion of the slice boundary. The projected edges are then combined and the interior edges are removed to make the final profile. 4) Trimming of the profiles to remove dangling edges and ensure that the geometry is closed and manifold. 5) Extrusion of the projected profiles along slicing direction.

[t!] [1] $O, S, n, V$ Squashed partial section of manifold shape $s_{n} \leftarrow$ Duplicate S byDirection $\mathrm{V}$

$\mathrm{I}_{n} \leftarrow \mathrm{O} \cap \mathrm{s}_{n}$

$P_{n} \leftarrow$ Project $\mathrm{I}_{n}$ to $S$

$P_{n}$ : non-manifold interior edge exist Trim interior edge unconnected edges Combine edge 
Table 1: Notation.

\begin{tabular}{ll}
\hline$O$ & Original Shape \\
$S$ & Solid plane \\
$n$ & Number of Slice \\
$V$ & Slicing Direction \\
$s_{n}$ & Duplicate $S, n$ times by $V$ direction \\
$I_{n}$ & Intersection Part of $O$ and $s_{n}\left(O \cap s_{n}\right)$ \\
$P_{n}$ & Projection result of $I_{n}$ to $S$ \\
$E_{n}$ & Extrude $P_{n}$ by direction $V$ \\
\hline
\end{tabular}

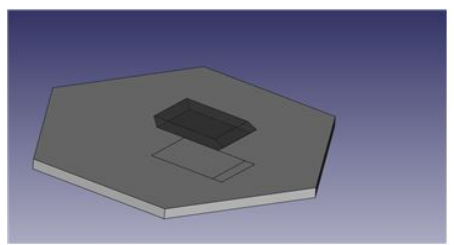

(a) Interior Edge

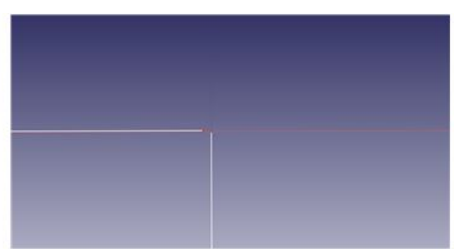

(b) Un-Connected Edge

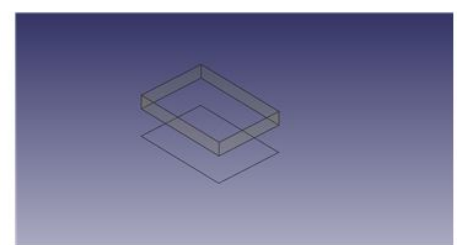

(c) Not Solid

Figure 13. Non-manifold Case.

$E_{n} \leftarrow$ Extrude $\mathrm{P}_{n}$ Shift $E_{n}$ by Direction $V+n$

\section{Case studies}

This section investigates the differences of representation methods in the design and manufacturing phases using STEP-NC additive manufacturing features. This section illustrates the differences and advantages of the proposed method which is defined in ISO 14649-17 [? ] among STEP-NC standardisation methods. The method presented in this paper is applied to multi-material shapes and squash slices to increase the accuracy in the geometry definition process. In particular, this section shows data loss and changes in accuracy through manufacturing phases from computer-aided design tools and process planning tools, like CAM systems, in order to show the verification and comparison. In the design phase, the accuracy and data size of additive shapes are analysed and, in particular, representation methods for controlling distributions to multi-colour are compared. In the process planning phase, the accuracy of the geometric operation of slicing algorithm is investigated in terms of missing volume and additional volume.

The case study is performed with different shapes so that individual functions highlight effective characteristics:

1. Comparison of two shapes for the distribution of two materials

2. Comparison of the volume of missing and additional volume according to slicing in different directions for curved features

3. Example of slicing column geometry with cooling channels

The quantitative comparison of the proposed method above verifies the differences in function.

\subsection{Gradient distribution}

NURBS was applied to two different 2D shapes in order to show the distribution of two colours. Figure 14 of two shapes shows the benefits are carried out of proposed methods. Examples are the front of a wardrobe and a curved beam to show the variety of AM process. These shapes are composed of different gradient colour changes from pink to blue. The top-left is the wardrobe coloured by simple distribution from top to 


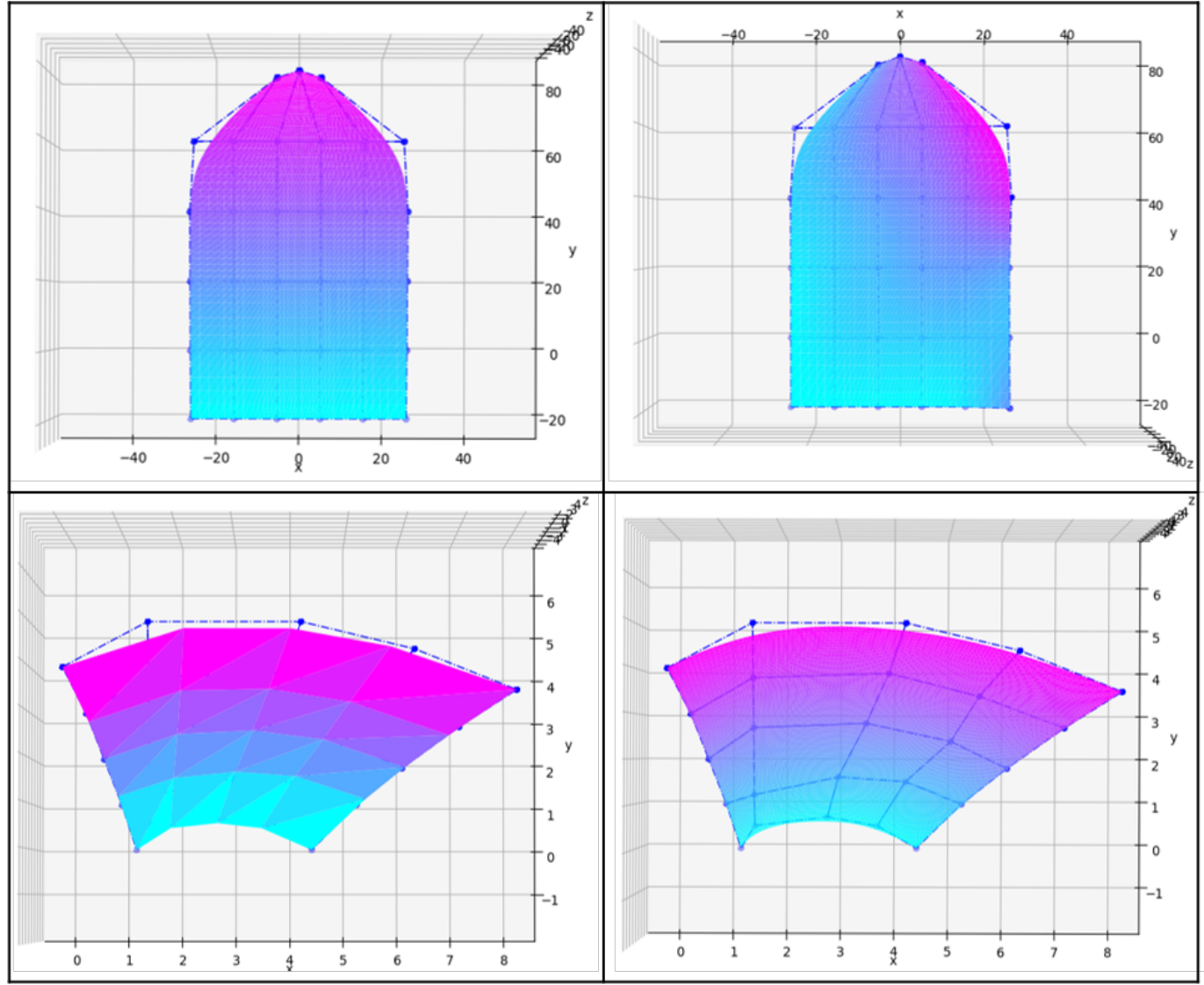

Figure 14. Gradient distribution of NURBS function

bottom whereas top-right is coloured with the pink source coming from the top-right corner. The bottom-right shows a NURBS distribution with control points on curved beam while the bottom-left is the result of discretizing the cross-section of the beam shape. The representation of the NURBS distribution requires $5 \times 5$ control points, degree 2 in $u$ and v, 2 knot vectors consisting of 9 real numbers. Discretizing is done after the CAM system loads a STEP-NC file. Thus, a discretized shape, like sampling number, polygon or voxel size, is not necessary for storing geometric data based on STEP-NC.

\subsection{Squashing operation for curved part}

The goal of the second test problem is to show how different slice geometries affect the accuracy of the created object. In both cases the squashing algorithm is used to create slices to ensure that there is no missing material. For comparison, the traditional slicing process for the object is shown in Figure 15.

The input geometry is a curved beam. The geometry of this model is shown in Figure 16 top-left. The slicing algorithm was applied to a each layer, with the result shown in Figure 16 top-right. The slice lines due to intersection with the layer planes are shown in side view in Figure 16 bottom-left. The object which would result is shown in side view in Figure 16 bottom-right, with the target shape in darker grey and the layers as they would be in lighter grey.

Comparing Figure 15 with Figure 16 bottom right the difference between classic slicing (Figure 15) and thick slicing and squashing (Figure 16 bottom right) can be clearly seen. Except at the top, where the slice extends outside the object, the result material is inside the desired shape with traditional slicing whereas the desired shape is surrounded by deposited material with the slicing-and-squashing method. 
Table 2: Volume Interval

\begin{tabular}{|c|c|c|c|}
\hline Layer & Base & Legacy & $\begin{array}{c}\text { Missing(-)/ } \\
\text { Additional( }(+)\end{array}$ \\
\hline 1 & 11.46 & 0 & -11.46 \\
2 & 24.26 & 21.06 & -3.2 \\
3 & 31.17 & 27.52 & -3.65 \\
4 & 36.92 & 34.69 & -2.23 \\
5 & 22.03 & 35 & +12.97 \\
\hline
\end{tabular}

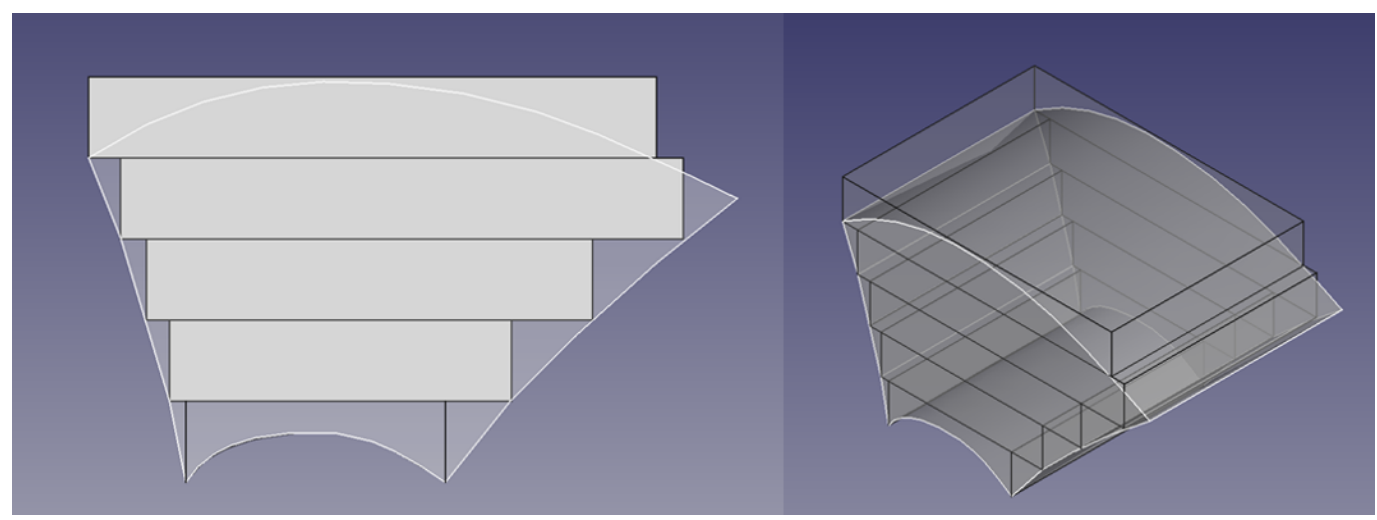

Figure 15. Legacy slicing

Table 3: Cylindrical slicing and squashing operation volume

\begin{tabular}{|c|c|c|c|}
\hline Base & $\begin{array}{c}\text { Legacy Slicing } \\
\text { (Fig 15) }\end{array}$ & $\begin{array}{c}\text { Squash Slicing } \\
\text { (Fig 16) }\end{array}$ & $\begin{array}{c}\text { Curved Slicing } \\
\text { (Fig 18) }\end{array}$ \\
\hline 125.84 & -718.27 & 161.11 & 153.96 \\
& $-7.57(-6 \%)$ & $+35.27(+28 \%)$ & $+28.12(+22 \%)$ \\
\hline
\end{tabular}

The squashing operation is important because it prevents missing material volumes and is consistent in adding material compared with traditional slicing. The squashing operation achieves consistency by combining the top and bottom sections together with silhouette geometry to calculate the material 'footprint' as the section profile. Tool path generation differentiates between two methods of offsetting each slice: direct offsetting and modified offsetting after each slice is squashed. The particular layers, such as bottom layer and top layer, seen in this comparison were chosen as they exhibit the highest missing volume of the slices. A comparison of the results produced by the legacy slicing operation is shown in Table 2. The results of the five layers are shown in Table 4 . The difference of volumes of original shape and the outputs of both algorithms is shown in Table 3. In Figure 16 bottom-right, it is clear that the proposed algorithm produces an output that is close to original shape and does not show any missing volume. 


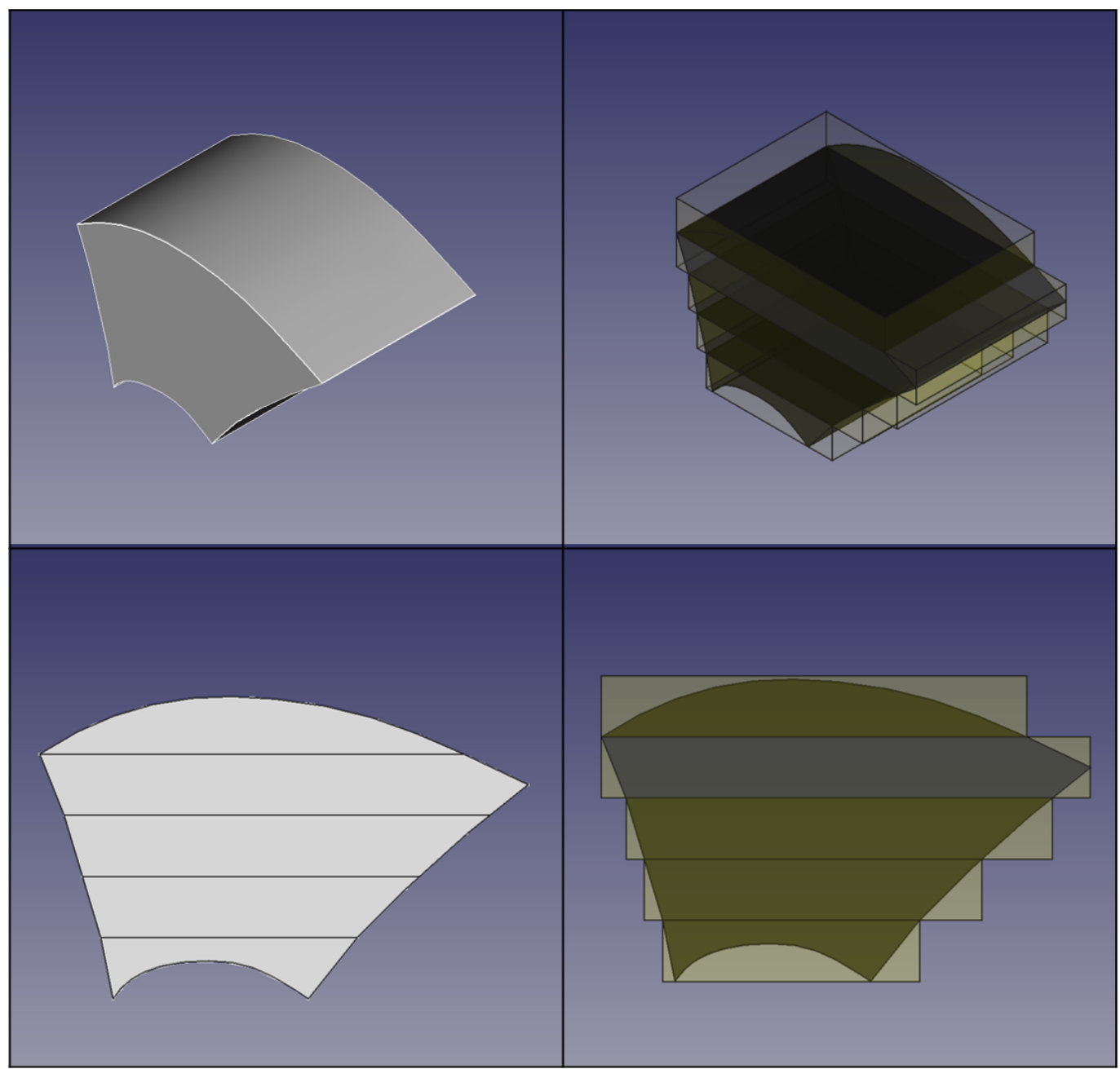

Figure 16. Result of squashing operation

Table 4: Comparison of Slicing method

\begin{tabular}{|c|cccccc|c|}
\hline $\begin{array}{c}\text { multirow Type } \\
\text { L1 }\end{array}$ & L2 & L3 & L4 & L5 & L6 & Sum \\
\hline $\begin{array}{c}\text { Legacy slicing } \\
\text { (Fig 15) }\end{array}$ & - & 21.06 & 27.52 & 34.69 & 35 & - & 118.27 \\
\hline $\begin{array}{c}\text { Squash slicing } \\
\text { (Fig 16) }\end{array}$ & 21 & 27.61 & 34.88 & 40 & 37.62 & - & 161.11 \\
\hline $\begin{array}{c}\text { Cur slicing } \\
\text { (Fig 18) }\end{array}$ & 19.3 & 24.32 & 29.22 & 34.68 & 41.08 & 5.36 & 153.96 \\
\hline
\end{tabular}

As well as missing volume, the gap resulting from slicing will be wider in the case of comparisons of shapes resulting from different file formats of STL and STEP-NC because slicing is performed on each profile which is extracted from the original shape represented by tessellated surface. The approaches to slice each layer reported recently are still aligned to direct slicing of tessellated shape even though Um and Stroud [8] already pointed out the issue of geometric accuracy. The challenge of achieving preservation of geometric accuracy imposes a significant hurdle to tool path generation of the additive process.

A related topic concerns the slicing with non-planar geometry. This is shown in Figure 17. The base shape is shown with the regular slice geometry. Figure 18 shows the results. The left side of the figure shows a side view of the shape with the with resulting slices and the right-hand side of the figure shows what the resulting figure would look like. 


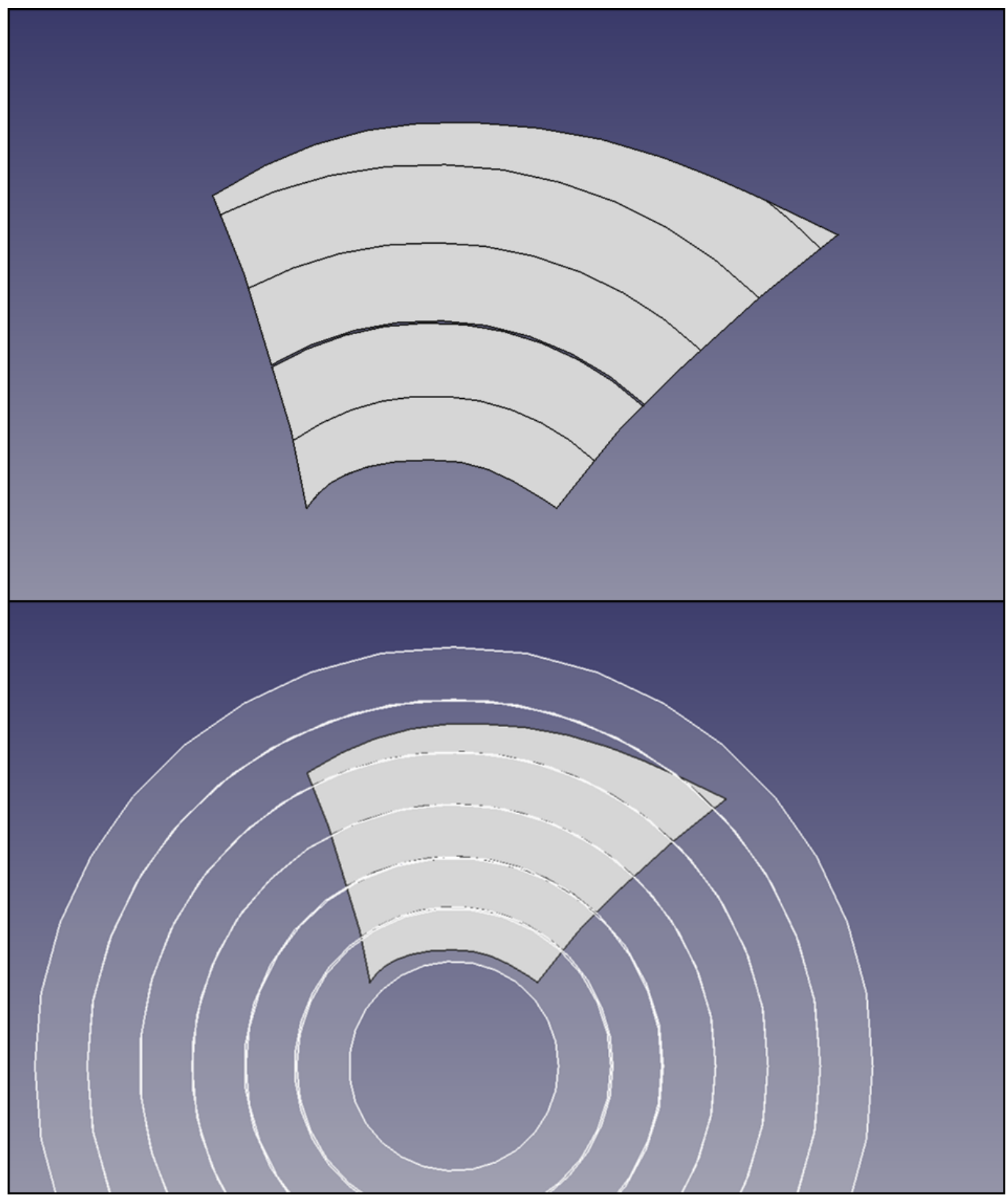

Figure 17. Curved slicing

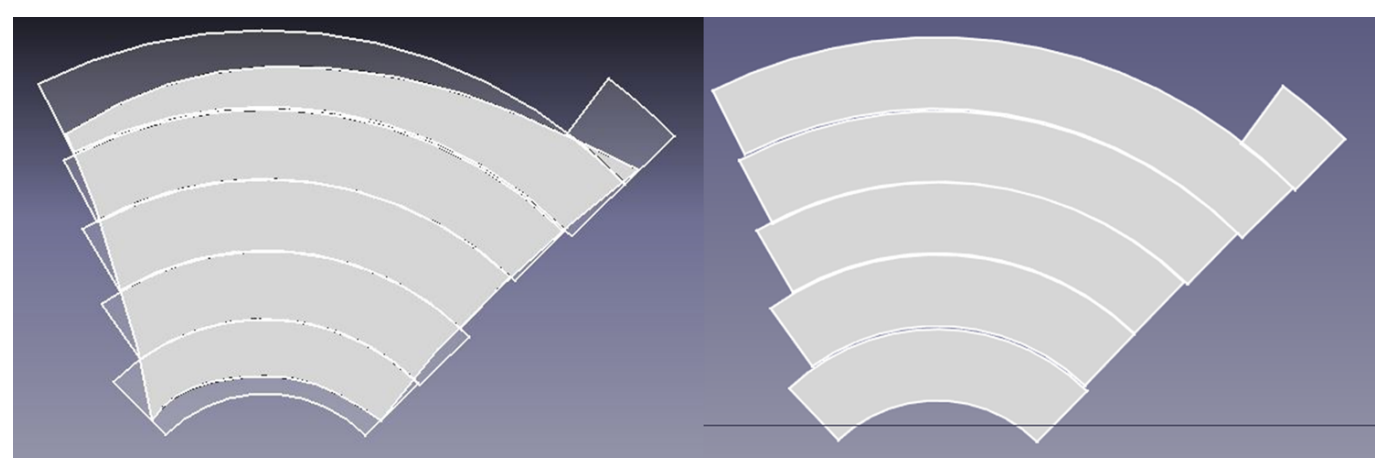

Figure 18. Cylindrical slicing and squashing operation

Circular slice geometry is used here to provide a simple comparison with planar geometry. Comparisons are given in Table 3. 


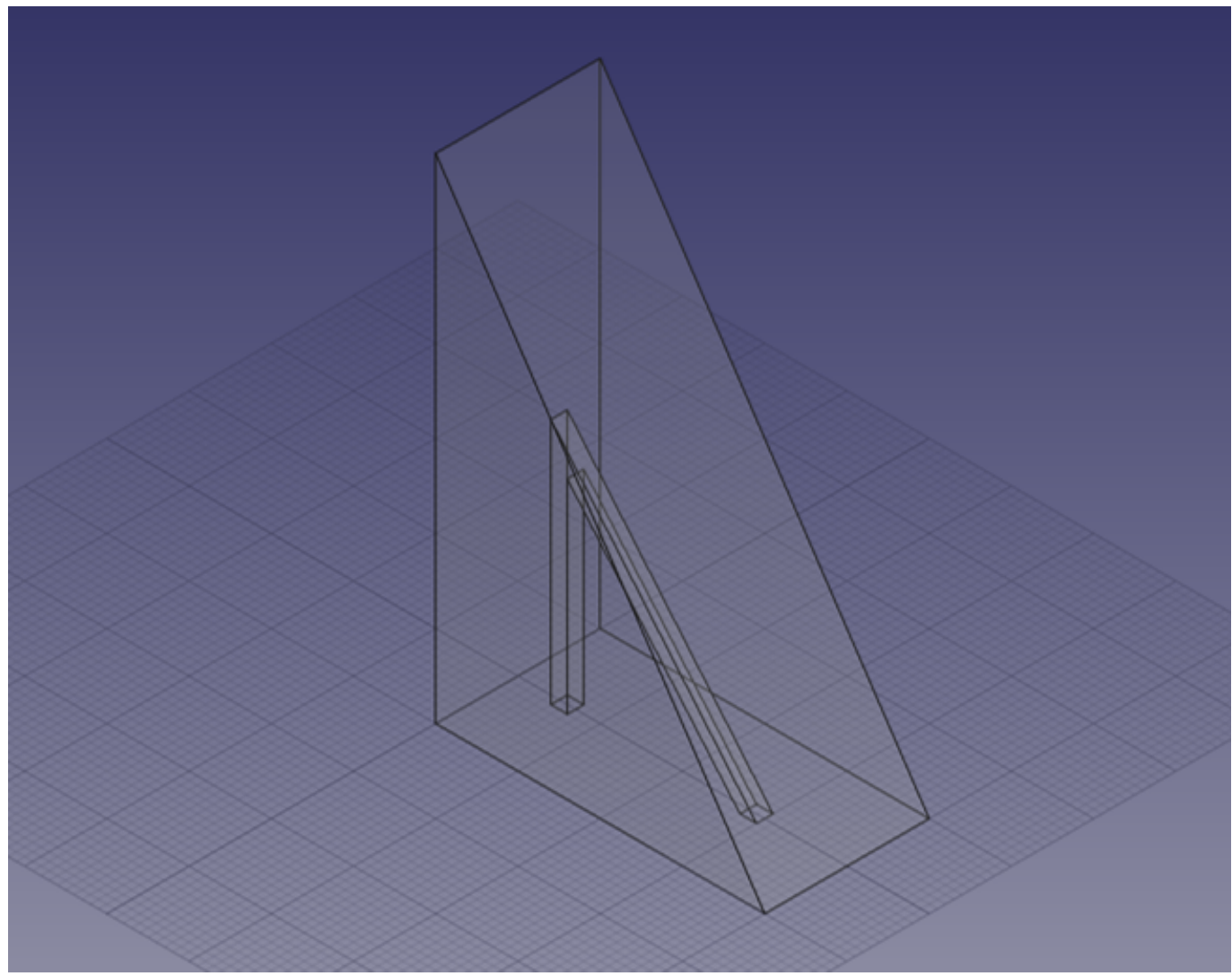

Figure 19. Inner micro structure

Different slice geometry is important in processes such as cladding, which is used for repair of existing objects, turbine blades, and for remanufacturing. To repair the leading edge of a turbine blade it is necessary for the additive process nozzle to be moved along a complex curved path aligned with the surface normal where the repair is being carried out. In this case, the depths of the sliced layers are not necessarily uniform and vary along the slope. This kind of process requires better geometric reasoning for something like squashing operation and exact rather than approximated object geometry.

\subsection{Squashing operation of empty material}

Additive manufacturing is widely thought of as capable of making any shape, however it is important to understand the limitations and implications. One of these concerns are internal micro-structures such as holes, internal filaments or porous structures. Because of the discrete nature of the additive process there is a limit on the micro-structure size. Figure 19 shows how a sloping filament might be treated with traditional slicing so that either the filament does not unite or the thickness is less than planned. Another interesting question concerns small holes. An example of an object with a small hole is shown in Figure 20. With traditional slicing the sloping hole is blocked because the single slice is extruded upwards, not at an angle, so the starts and ends of the hole sections do not meet as shown in Figure 20.

Normal squashing cannot help this problem because the material footprint would cover the hole. However, if the hole is defined as a core with empty material and if that material has a higher priority than the surrounding material, then the hole would consist of a set of connected cavities, as shown in Figure 21. Top left in the figure is the object and the slice planes, shown right with how the object would be cut and in side view bottom left. Bottom right in the figure shows the result with the square cavities. This is not what the designer may expect, though. 


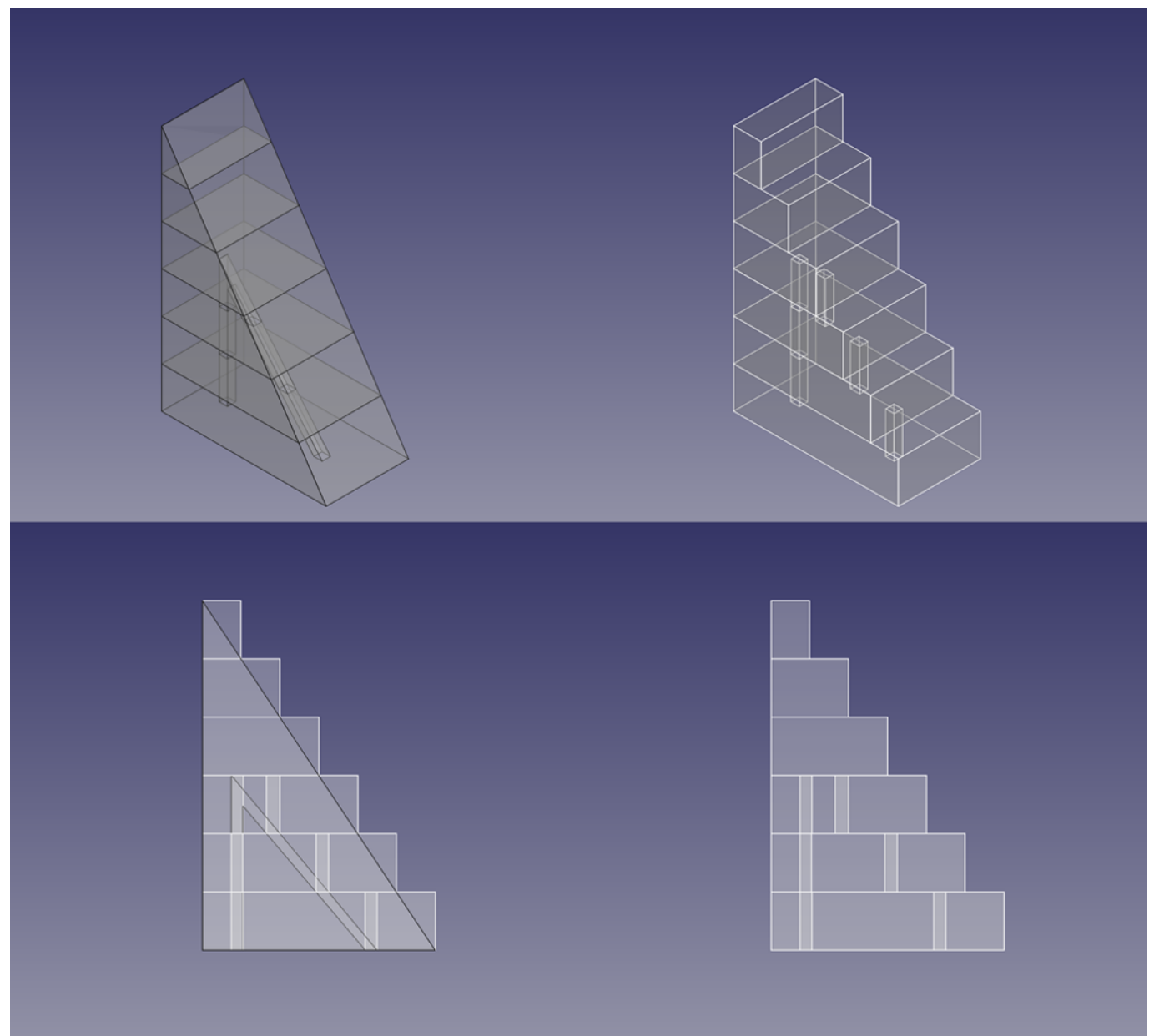

Figure 20. Legacy slice operation of inner micro structure

\section{Discussion}

From the result of gradient distribution along NURBS and of squash slicing operation, in the design phase and manufacturing phase mentioned earlier, the case study looks at the multi-material related cases and compares the differences in features that come out of a squash operation at the same time.

The gradient case study demonstrates that the use of NURBS to define colour gives good results. It is more efficient in terms of storage space than both voxelised and tessellated representations. The use of NURBS for multi-material definition has similar advantages. The second case study shows both the use of squashing to avoid missing volume in created shapes. This means that finishing processes such as milling or grinding can be used to remove extra material if necessary. The case study also shows the use of non-planar slicing techniques and quantifies the advantages for the object used. The use of non-planar slices is possible in some processes such as cladding. The third case study demonstrates the need for handling micro-holes in a special way using void material definitions.

\section{Conclusions}

STEP-NC based representation maintains accuracy throughout the whole additive process chain by using exact $\mathrm{CAD}$ data. New features and operations are required for this as part of the control, but since STEP-NC, ISO 14649-17, is a micro process plan, any manufacturing decisions are made by the manufacturer or by the machine itself. This means that the design information is used directly and the designer is not required to make manufacturing decisions. This paper concerns the new features and operations needed for 


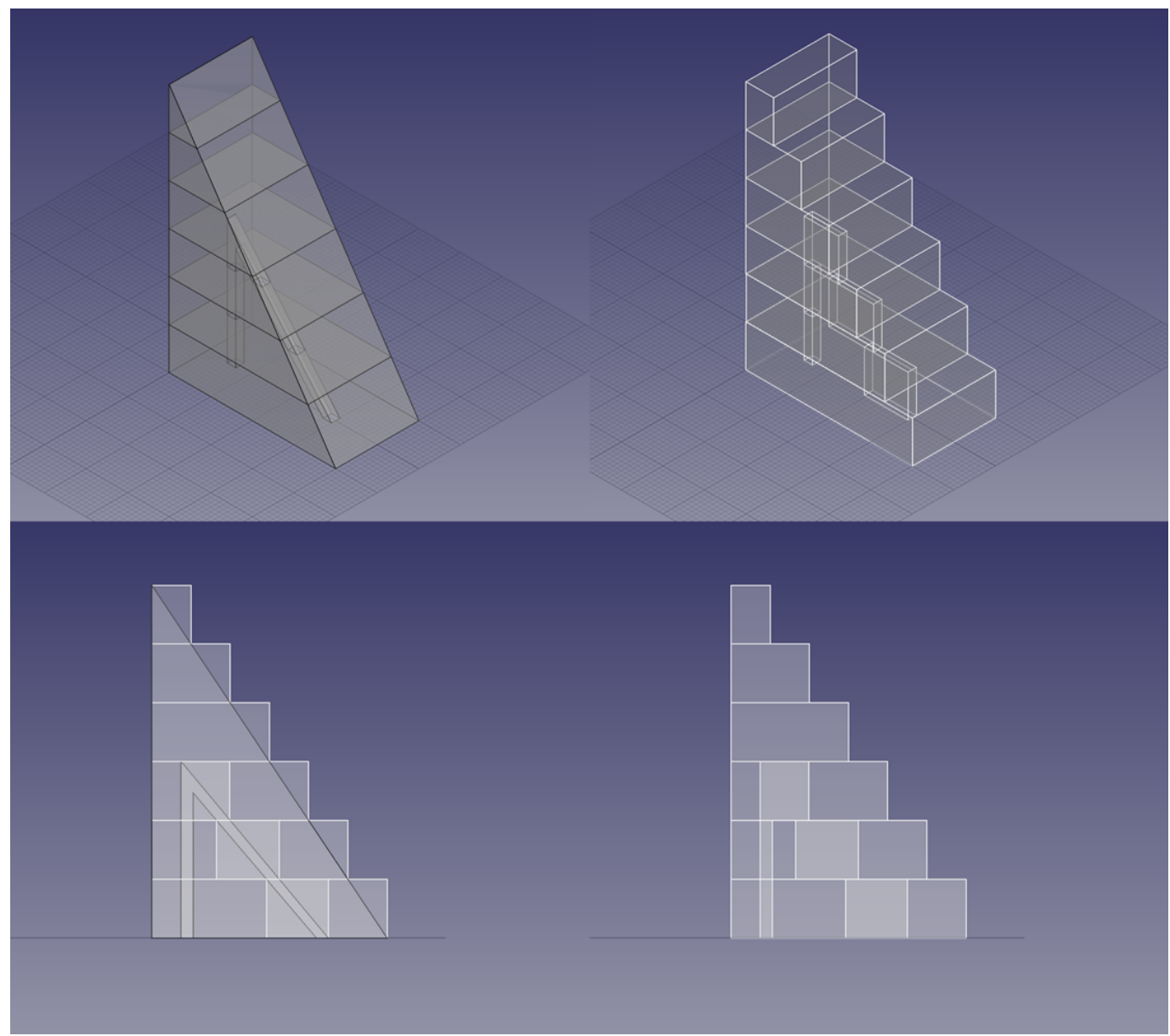

Figure 21. Squashing operation of inner micro structure

control to move additive manufacturing closer to being 'plug-and-play'.

The ISO 14649-17 definition covers the requirements of the designer and its use is described in this paper. Future work involves the development of a modern advanced controller which should be able to:

1. Slice an exact model, communicating the slices if necessary

2. Perform a limited set of solid operations

3. Monitor the process for adaptive control

Author Contributions: For research articles with several authors, a short paragraph specifying their individual contributions must be provided. The following statements should be used "Conceptualization, X.X. and Y.Y.; methodology, X.X.; software, X.X.; validation, X.X., Y.Y. and Z.Z.; formal analysis, X.X.; investigation, X.X.; resources, X.X.; data curation, X.X.; writing-original draft preparation, X.X.; writing-review and editing, X.X.; visualization, X.X.; supervision, X.X.; project administration, X.X.; funding acquisition, Y.Y. All authors have read and agreed to the published version of the manuscript.", please turn to the CRediT taxonomy for the term explanation. Authorship must be limited to those who have contributed substantially to the work reported.

Funding: Please add: "This research received no external funding" or "This research was funded by NAME OF FUNDER grant number XXX." and and "The APC was funded by XXX". Check carefully that the details given are accurate and use the standard spelling of funding agency names at https:/ / search.crossref.org/funding, any errors may affect your future funding.

Acknowledgments: This work was supported by the National Research Foundation of Korea(NRF) grant funded by the Korea government(MEST) (No. NRF-2019R1G1A1100471) 
authors declared no potential conflicts of interest with respect to the research, authorship, and/or publication of this article.

Sample Availability: Samples of the compounds ... are available from the authors.

\section{References}

1. Bonnard, R. Proposition de chaine numerique pour la fabrication additive. Doctoral dissertation, Ecole Centrale de Nantes (ECN) 2010.

2. Roscoe, L.E. Stereolithography interface specification. America-3D Systems Inc 1988, 27, 10.

3. Carleberg, P. Product model driven direct manufacturing. International Solid Freeform Fabrication Symposium 1994.

4. Industrial Automation Systems and Integration-Product Data Representation and Exchange-AP242 managed model based 3D engineering. Standard ISO 10303-242, International Standard Organization, Geneva, Switzerland., 2014.

5. Hiller, J.D.; Lipson, H. STL 2.0: a proposal for a universal multi-material additive manufacturing file format. Proceedings of the Solid Freeform Fabrication Symposium, 2006, Vol. 3, pp. 266-278.

6. Le Bourhis, F.; Kerbrat, O.; Hascoet, J.Y.; Mognol, P. Sustainable manufacturing: evaluation and modeling of environmental impacts in additive manufacturing. International Journal of Advanced Manufacturing Technology 2013, 69(9-12), 1927-1939.

7. Bonnard, R.; Hascoët, J.Y.; Mognol, P.; Zancul, E.; Alvares, A.J. Hierarchical object-oriented model (HOOM) for additive manufacturing digital thread. Journal of Manufacturing Systems 2019, 50, 36-52.

8. Um, J.Y.; Rauch, M.; Hascoet, J.Y.; Stroud, I. STEP-NC compliant process planning of additive manufacturing: remanufacturing. The International Journal of Advanced Manufacturing Technology 2017, 88(5-8), 1215-1230.

9. Newman, S.; Zhu, Z.; Dhokia, V.; Shokrani. Process planning for additive and subtractive manufacturing technologies. In CIRP Annals 2015, 64(1), 467-470.

10. Flynn, J.M.; Shokrani, A.; Newman, S.T.; Dhokia, V. Hybrid additive and subtractive machine tools-Research and industrial developments. International Journal of Machine Tools and Manufacture 2016, 101, 79-101.

11. Zhu, Z.; Dhokia, V.G.; Nassehi, A.; Newman, S.T. A review of hybrid manufacturing processes-state of the art and future perspectives. International Journal of Computer Integrated Manufacturing 2013, 26(7), 596-615.

12. Lee, B.N.; Pei, E.; Um, J. An overview of information technology standardization activities related to additive manufacturing. Progress in Additive Manufacturing 2019, 4(3), 345-354.

13. Pei, E.; Loh, G.H. Technological considerations for 4D printing: an overview. Progress in Additive Manufacturing 2018, 3(1-2), 95-107.

14. Industrial automation systems and integration - Physical device control - Data model for computerized numerical controllers - Part 10: General process data. Standard ISO 14649-10, International Standard Organization, Geneva, Switzerland., 2004.

15. Vaezi, M.; Chianrabutra, S.; Mellor, B.; Yang, S. Multiple material additive manufacturing - Part 1: a review: this review paper covers a decade of research on multiple material additive manufacturing technologies which can produce complex geometry parts with different materials. Virtual and Physical Prototyping 2013, 8(1), 19-50.

16. Kokkinis, D.; Schaffner, M.; Studart, A.R. Multimaterial magnetically assisted 3D printing of composite materials. Nature communications 2015, 6(1), 1-10.

17. Pa, P.; Larimore, Z.; Parsons, P.; Mirotznik, M. Multi-material additive manufacturing of embedded low-profile antennas. Electronics Letters 2015, 51(20), 1561-1562.

18. Ge, Q.; Sakhaei, A.H.; Lee, H.; Dunn, C.K.; Fang, N.X.; Dunn, M.L. Data model for additive manufacturing digital thread: state of the art and perspectives. Scientific Reports 2016, 6(1).

19. Liu, Z.H.; Zhang, D.Q.; Sing, S.L.; Chua, C.K.; Loh, L.E. Interfacial characterization of SLM parts in multi-material processing: Metallurgical diffusion between 316L stainless steel and C18400 copper alloy. Materials Characterization 2014, 94, 116-125.

20. Gupta, V.; Tandon, P. Heterogeneous object modeling with material convolution surfaces. Computer-Aided Design 2015, 62, 236-247.

21. Kumar, V.; Dutta, D. An assessment of data formats for layered manufacturing. Advanced Engineering Software 1997, 28(3), 151-164.

22. Pratt, M.J.; Bhatt, A.D.; Dutta, D.; Lyons, K.W.; Patil, L.; Sriram, R.D. Progress towards an international standard for data transfer in rapid prototyping and layered manufacturing. Computer-Aided Design 2002, 34(14), 1111-1121. 
23. Wang, W.M.; Zanni, C.; Kobbelt, L. Improved surface quality in 3D printing by optimizing the printing direction. Virtual and Physical Prototyping 2016, 35(2), 59-70.

24. Mohammed, M.; Tatineni, J.; Cadd, B.; Peart, P.; Gibson, I. Applications of 3D topography scanning and multi-material additive manufacturing for facial prosthesis development and production. Proceedings of the 27th Annual International Solid Freeform Fabrication Symposium, 2016, pp. 1695-1707.

25. Bonnard, R.; Hascoet, J.Y.; Mognol, P.; Stroud, I. STEP-NC digital thread for additive manufacturing: data model, implementation and validation. International Journal of Computer Integrated Manufacturing 2018, 31(11), 1141-1160.

26. Bonnard, R.; Hascoet, J.Y.; Mognol, P. Data model for additive manufacturing digital thread: state of the art and perspectives. International Journal of Computer Integrated Manufacturing 2019, 32(12), 1170-1191.

27. Zhao, G.; Cao, X.; Xiao, W.; Liu, Q.; Jun, M.B.G. STEP-NC feature-oriented high-efficient CNC machining simulation. International Journal of Advanced Manufacturing Technology 2020, 106(5), 2363-2375.

28. Lee, K.; Jee, H. Slicing algorithms for multi-axis 3-D metal printing of overhangs. Journal of Mechanical Science and Technology 2015, 29(12), 5139-5144.

29. Steuben, J.C.; Iliopoulos, A.P.; Michopoulos, J.G. Implicit slicing for functionally tailored additive manufacturing. Computer-Aided Design 2016, 77, 107-119.

30. Wu, J.; Wang, C.C.; Zhang, X.; Westermann, R. Self-supporting rhombic infill structures for additive manufacturing. Computer-Aided Deisgn 2016, 80, 32-42. 
